\title{
Response Surface Methodology of Alcohol Production from TWR Sugar using Saccharomyces cerevisiae TISTR 5339
}

\author{
Athitan Timyamprasert ${ }^{1 *}$, Thanit Vairojanawong ${ }^{1}$ and Siritorn Teeravet $^{2}$ \\ ${ }^{1}$ Expert Centre of Innovative Industrial Robotics and Automation, Thailand Institute of Scientific and Technological Research (TISTR), \\ Khlong Ha, Khlong-Luang, Pathumthani, 12120, Thailand \\ ${ }^{2}$ Biodivesity Research Centre, Thailand Institute of Scientific and Technological Research (TISTR), Khlong Ha, Khlong-Luang, \\ Pathumthani, 12120, Thailand
}

\begin{abstract}
The aim of this research was to develop technique to produce alcohol from Thai white rice (TWR) using Saccharomyces cerevisiae TISTR 5339. TWR sugar was produced from TWR with Amylomyces rouxii TISTR 3182. Response surface methodology (RSM) was applied for investigating the experimental design for production the alcohol. There were 20 experiments involving the three investigated variables of Saccharomyces cerevisiae TISTR 5339 to amount of TWR sugar ratio, concentration of TWR sugar (\% Brix) and retention time of fermentation that were studied on alcohol to optimize the condition for production the alcohol. Design of experiment was performed by application of 5-level-3-factors central composite design in order to study the optimum condition for production the alcohol. The investigated results showed that the optimum condition was $4.0 \mathrm{ml}$ of Saccharomyces cerevisiae TISTR 5339 amount to $100 \mathrm{ml}$ of TWR sugar amount, 20\%Brix (concentration of TWR sugar) and 7 days of fermentation time.
\end{abstract}

\section{Introduction}

In Thailand, large quantities of Thai white rice (TWR) are consumed as principal food for daily meals in the north, south and central parts of the country. The central and north regions are also the main cultivating sources of the TWR. The variety of TWR is mainly composed of starch which constitutes nearly $90 \%$ of the dry matter when milled [1]. Further, the amylose content is about $2.39 \pm$ $0.25 \%$ of the starch [2]. The nature of cooked TWR is soft, so it can be used as the raw materials for producing alcohol to make vinegar, drinks and fuel. In this study, sugar from TWR was used as raw materials for producing the alcohol by using Saccharomyces cerevisiae TISTR 5339 and TWR sugar. In this process of production, the cooked TWR was fermented with Amylomyces rouxii TISTR 3182 for producing the TWR sugar and then separating the TWR sugar to mix with Saccharomyces cerevisiae TISTR 5339 for producing the alcohol. Therefore, the objective of this study was to investigate the effect of Saccharomyces cerevisiae TISTR 5339 to amount of Thai white rice sugar ratio, concentration of Thai white rice sugar (\%Brix) and retention time of fermentation on the amount of alcohol for optimizing condition of producing the alcohol from TWR sugar.

RSM (Response surface methodology) is a useful statistical technique which has been applied in research into complex variable process. The multiple regression and correlation analyses are used as tools to assess the effect of two or three independent factors on the dependent variables. In addition, the CCD (Central composite design) of RSM has been applied in the optimization of several biotechnological and chemical processes. The principle advantage of RSM is the reduced number of experimental runs required to generate sufficient information for a statistically acceptable result. RSM has been successfully applied in the study and optimization of parameters for fermentation, rice sugar, enzyme and alcohol production $[3,4,5,6]$. In this work, the TWR sugar was used as material for production the alcohol by using Saccharomyces cerevisiae TISTR 5339 to study the optimized condition by RSM to produce the alcohol.

\section{Materials and Methods}

\subsection{Materials}

Thai white rice (TWR) was obtained from a market in Pathumthani province, Thailand. Saccharomyces cerevisiae TISTR 5339 and Amylomyces rouxii TISTR 3182 were collected from TISTR Culture Collection Centre of Biodivesity Research Centre, Thailand Institute of Scientific and Technological Research (TISTR) in Thailand.

\subsection{Preparation for material}

The $1.00 \mathrm{~kg}$ of the TWR was washed with water about 23 times, and then autoclaved at $15 \mathrm{Ib} / \mathrm{in}^{2}$ at $121^{\circ} \mathrm{C}, 15 \mathrm{~min}$.

${ }^{*}$ Corresponding author's e-mail: athitan@tistr.or.th 
Apparatus and the fermentation for producing the TWR sugar was conducted in a stainless steel basket of $1000 \mathrm{ml}$ jar equipped with a lid and a $0.3 \mu \mathrm{m}$ ceramic filter for filtering to clean inlet air. At the bottom of the jar was designed and made a hole equipped with a stopcock for collecting the TWR sugar. This jar was sterilized by autoclave at $15 \mathrm{Ib} / \mathrm{in}^{2}$ at $121^{\circ} \mathrm{C}, 15 \mathrm{~min}$. For fermentation, The $500 \mathrm{~g}$ of sterilized TWR was mixed with a certain quantity of Amylomyces rouxii TISTR 3182, and then $5 \mathrm{ml}$ of sterilized water was added and transferred to the stainless steel basket in the jar for fermentation at room temperature. The TWR sugar was used as the raw materials for producing alcohol.

\subsection{Apparatus and Fermentation Procedures}

For producing the alcohol, the $100 \mathrm{ml}$ of sterilized TWR sugar was mixed with a certain quantity of Saccharomyces cerevisiae TISTR 5339 in $250 \mathrm{ml}$ of volumetric flash with

Table1. Independent variables and levels used for CCD for producing alcohol from TWR sugar.

\begin{tabular}{|c|c|c|c|c|c|c|}
\hline \multirow{3}{*}{ Variables } & \multirow[b]{2}{*}{ Symbols } & \multicolumn{5}{|c|}{ Code levels $^{\mathrm{a}}$} \\
\hline & & -1.68 & -1 & 0 & +1 & +1.68 \\
\hline & (Uncoded) & $(-\alpha)$ & & & & $(+\alpha)$ \\
\hline $\begin{array}{l}\text { Saccharomyces cerevisiae TISTR } 5339 \\
\text { to amount of TWR sugar ratio }(\mathrm{ml} / \mathrm{ml})\end{array}$ & $\mathrm{S}$ & 0.96 & 3 & 6 & 9 & 11.04 \\
\hline Concentration of TWR sugar (\% Brix) & $\mathrm{C}$ & 14.96 & 17 & 20 & 23 & 25.04 \\
\hline Retention time of fermentation (day) & $\mathrm{T}$ & 1.12 & 3.5 & 7 & 10.5 & 12.88 \\
\hline
\end{tabular}

\subsection{Statistical Analysis}

The experimental data of the production of alcohol from TWR sugar using Saccharomyces cerevisiae TISTR 5339 was performed by the RSM that following a second-order polynomial equation generated SPSS (Statistical package for social science) software. The suitable model was used to generate a response surface plot by STATISTICA software. The quadratic response surface model was shown in equation (1).

$$
Y=\beta_{o}+\sum_{i=1}^{3} \beta_{i} X_{i}+\sum_{i=1}^{3} \beta_{i i} X_{i}^{2}+\sum_{i=1}^{2} \sum_{i=j+1}^{2} \beta_{i j} X_{i} X_{j}
$$

Where $Y$ is the response (amount of alcohol (\%)), $\beta_{0}$ is constant, $X_{i}$ and $X_{j}$ are the independent variables, $\beta_{I}$ and $\beta_{j}$ are the linear term coefficients, $\beta_{i i}$ is quadratic term coefficients, and $\beta_{i j}$ is the interaction constant coefficients. SPSS package was used for regression ANOVA (analysis of variance) and RSM was performed using the SPSS software [7].

\section{Results and Discussion}

\subsection{Optimization of production of alcohol from TWR sugar}

The design points of the amount of alcohol (\%) used CCD arrangement and response for production of alcohol from a lid at room temperature. After that the fermentation was started and timed immediately.

\subsection{Experimental Design for production of alcohol from TWR sugar}

A five-level and three-factor CCD with 20 experiments was employed in this study. Saccharomyces cerevisiae TISTR 5339 to amount of TWR sugar ratio (S), concentration of TWR sugar (\% Brix) (C) and retention time of fermentation $(\mathrm{T})$ were the independent variables to optimize the production of alcohol from TWR sugar. The coded and uncoded levels of the independent variables, independent factor, levels and experimental design were shown in table1. The central values (zero level) chosen for experimental design were $6.0(\mathrm{ml})$ of Saccharomyces cerevisiae TISTR 5339 amount, 20 (\% Brix) of TWR sugar and 7.0 days of fermentation time.
TWR sugar using Saccharomyces cerevisiae TISTR 5339 were shown in table 3 . In tables 2 and 4, statistical analysis of the model was performed to determine the variance (ANOVA) for the quadratic polynomial mode land linear regression coefficients indicated that were obtained by employing a least square technique to predict quadratic polynomial model for amount of alcohol (\%).

The characteristics of the models and the coefficients indicated that the predictability of the model was at $95 \%$ confidence level. The model had high correlation coefficient, a significant F-value, an insignificant lack-offit F-value. The analysis of variance (ANOVA) showed that the model F-values of 41.639 with significance was 0.000 which less than 0.05 for the models, implied the model was significant. The high correlation coefficient $\left(\mathrm{R}^{2}=0.974\right)$ indicated that the model was suitable to represent the real relationships among the parameters studied. The insignificant lack-of-fit which is relative to the pure error of the experiments also indicates that the models were suitable to represent the experimental data. Coefficients of full model equation were evaluated by regression analysis and tested for their significances. The models to predict the amount of alcohol (\%) of production of alcohol from TWR sugar using Saccharomyces cerevisiae TISTR 5339 was presented in equation (2).

$$
\mathrm{Y}=-54.538+1.534 \mathrm{~S}+4.833 \mathrm{C}+2.299 \mathrm{~T}-0.076 \mathrm{~S}^{2} \text { - }
$$$$
0.115 \mathrm{C}^{2}
$$$$
-0.114 \mathrm{~T}^{2}-0.021 \mathrm{SC}-0.006 \mathrm{CT}+0.006 \mathrm{ST}
$$ 
Table2. Analysis of variance (ANOVA) for the quadratic polynomial model for producing alcohol from TWR sugar.

\begin{tabular}{llllll}
\hline Model & $\begin{array}{l}\text { Sum of } \\
\text { squares }\end{array}$ & df & Mean square & F & Sig. \\
\hline Regression & 116.820 & 9 & 12.980 & 41.639 & $0.000^{\mathrm{a}}$ \\
Residual & 3.117 & 10 & 0.312 & & \\
Total & 119.937 & 19 & & & \\
\hline a Predictors: (Constant) ST, CC, TT, SS. SC, CT, S, T, C & & &
\end{tabular}

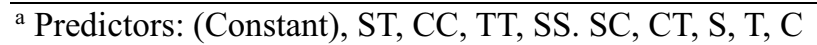

Table3. Central composite design (CCD) arrangement and response for producing alcohol from TWR sugar.

\begin{tabular}{lllcccccc}
\hline Treatment & $\mathrm{S}$ & $\mathrm{C}$ & $\mathrm{T}$ & \multicolumn{2}{c}{$\mathrm{S}$} & $\mathrm{C}$ & $\mathrm{T}$ & \multicolumn{2}{c}{ Alcohol (\%) } \\
\cline { 5 - 8 } & & & & $(\mathrm{ml} / \mathrm{ml})$ & $(\%$ Brix) & $($ day) & Experimental & Predicted \\
\hline 1 & -1 & -1 & -1 & 3.00 & 17.00 & 3.50 & 4.00 & 3.59 \\
2 & -1 & -1 & 1 & 3.00 & 17.00 & 10.50 & 8.00 & 7.92 \\
3 & -1 & 1 & -1 & 3.00 & 23.00 & 3.50 & 5.00 & 4.49 \\
4 & -1 & 1 & 1 & 3.00 & 23.00 & 10.50 & 9.00 & 8.57 \\
5 & 1 & -1 & -1 & 9.00 & 17.00 & 3.50 & 5.00 & 5.31 \\
6 & 1 & -1 & 1 & 9.00 & 17.00 & 10.50 & 9.50 & 9.89 \\
7 & 1 & 1 & -1 & 9.00 & 23.00 & 3.50 & 5.50 & 5.44 \\
8 & 1 & 1 & 1 & 9.00 & 23.00 & 10.50 & 9.50 & 9.78 \\
9 & -1.68 & 0 & 0 & 0.96 & 20.00 & 7.00 & 6.00 & 6.83 \\
10 & +1.68 & 0 & 0 & 11.04 & 20.00 & 7.00 & 10.00 & 9.29 \\
11 & 0 & -1.68 & 0 & 6.00 & 14.96 & 7.00 & 7.00 & 6.74 \\
12 & 0 & +1.68 & 0 & 6.00 & 25.04 & 7.00 & 7.00 & 7.40 \\
13 & 0 & 0 & -1.68 & 6.00 & 20.00 & 1.12 & 2.00 & 2.41 \\
14 & 0 & 0 & +1.68 & 6.00 & 20.00 & 12.88 & 10.00 & 9.69 \\
15 & 0 & 0 & 0 & 6.00 & 20.00 & 7.00 & 10.00 & 9.99 \\
16 & 0 & 0 & 0 & 6.00 & 20.00 & 7.00 & 9.50 & 9.99 \\
17 & 0 & 0 & 0 & 6.00 & 20.00 & 7.00 & 10.00 & 9.99 \\
18 & 0 & 0 & 0 & 6.00 & 20.00 & 7.00 & 10.50 & 9.99 \\
19 & 0 & 0 & 0 & 6.00 & 20.00 & 7.00 & 10.00 & 9.99 \\
20 & 0 & 0 & 0 & 6.00 & 20.00 & 7.00 & 10.00 & 9.99 \\
\hline
\end{tabular}

Table4. Regression coefficients of predicted quadratic polynomial model for the regression equation of alcohol production from

\begin{tabular}{cccccc}
\hline \multirow{2}{*}{ Model } & \multicolumn{6}{c}{ TWR sugar. } \\
& Unstandardized Coefficients & $\begin{array}{c}\text { Standardized } \\
\text { Coefficients }\end{array}$ & t & Sig. \\
\cline { 2 - 6 } & B & Std. error & Beta & -7.099 & 0.000 \\
\hline (Constant) & -54.538 & 7.682 & & 3.062 & 0.012 \\
S & 1.534 & 0.501 & 1.552 & 7.081 & 0.000 \\
C & 4.833 & 0.683 & 4.890 & 5.356 & 0.000 \\
T & 2.299 & 0.429 & 2.714 & -4.629 & 0.001 \\
SS & -0.076 & 0.016 & -0.950 & -7.034 & 0.000 \\
CC & -0.115 & 0.016 & -4.674 & -9.439 & 0.000 \\
TT & -0.114 & 0.012 & -1.938 & -0.950 & 0.365 \\
SC & -0.021 & 0.022 & -0.443 & -0.317 & 0.758 \\
CT & -0.006 & 0.019 & -0.148 & 0.317 & 0.758 \\
ST & 0.006 & 0.019 & 0.062 & & \\
\hline
\end{tabular}

\subsection{Response Surface Plots of production of alcohol from TWR sugar}

The optimized levels of variables were determined by constructing three-dimensional surface plots according to equation (2). The figure 1(a) shows the effect of Saccharomyces cerevisiae TISTR 5339 to amount of Thai white rice sugar ratio $(\mathrm{S})$, concentration of Thai white rice sugar (\%Brix) (C) on the alcohol amount (\%) of production of alcohol from TWR sugar and their mutual interaction on the alcohol amount (\%). The figure 1(b) 
shows the effect of Saccharomyces cerevisiae TISTR 5339 to amount of Thai white rice sugar ratio, retention time of fermentation $(\mathrm{T})$ on the alcohol amount $(\%)$ and the figure 1(c) shows the effects of concentration of Thai white rice sugar (\%Brix) and retention time of fermentation on the alcohol amount (\%). The figure 1(a), 1(b), 1(c) presented the limit values of the optimum values in producing the alcohol (\%). They indicate that concentration of Thai white rice sugar is $18-23 \%$ of Brix and 7-11 days of fermentation time. Saccharomyces cerevisiae TISTR 5339 amount should be used between 4.0 to $8.0 \mathrm{ml}$ to $100 \mathrm{ml}$ of Thai white rice sugar amount which is obtained from the RSM.

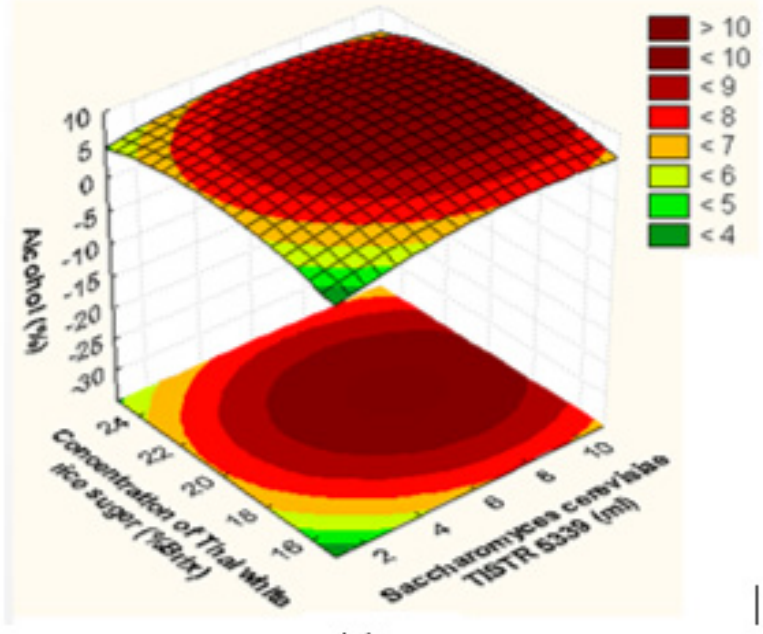

(a)

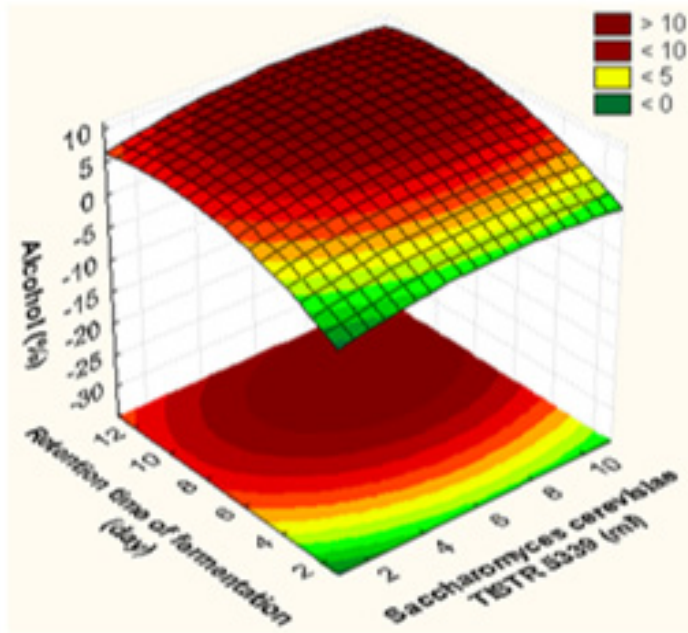

(b)

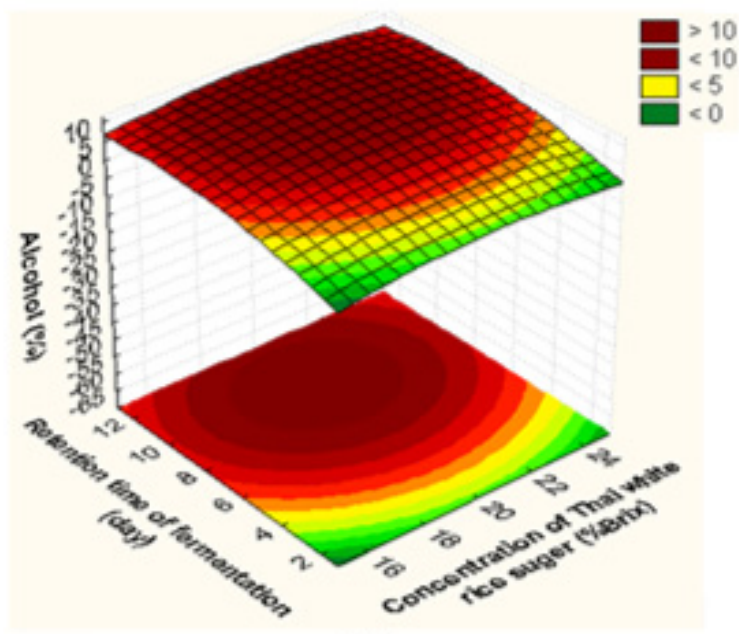

(c)

Figure 1. Response surface plots representing the effect of factors on alcohol predicted from the quadratic polynomial model.

\subsection{Model Development for production of alcohol from TWR sugar}

The model was developed to predict optimization of production of alcohol from TWR sugar with Saccharomyces cerevisiae TISTR 5339 to amount of TWR sugar ratio and concentration of TWR sugar (\%Brix) by using optimization function of the SPSS software. The five conditions were selected from the optimum values in producing the alcohol from response surface plot figure 1 . They were both experimental values and predicted values which indicated that the comparison of both value was good correspondence between them, the empirical models derived from RSM can be used to describe the relationship between the factors and response in producing the alcohol from TWR sugar. The prediction of the developed model showed that the optimized condition at $4.0 \mathrm{ml}$ of
Saccharomyces cerevisiae TISTR 5339 amount to $100 \mathrm{ml}$ of TWR sugar amount, $20 \%$ Brix of TWR sugar for 7 days of fermentation time could produce the alcohol to $10.00 \%$.

\section{Conclusions}

The TWR could be used as raw material to produce alcohol. The cooked TWR can be fermented with Amylomyces rouxii TISTR 3182 to produce the TWR sugar. This TWR sugar can be used as the raw material for producing alcohol. The optimum conditions of alcohol production from the TWR sugar were obtained at $4.0 \mathrm{ml}$ of Saccharomyces cerevisiae TISTR 5339 amount to 100 $\mathrm{ml}$ of TWR sugar amount, $20 \%$ Brix of TWR sugar for 7 days of fermentation time. This condition was selected as the optimum condition because it could produce the alcohol in minimum amount of Saccharomyces cerevisiae 
TISTR 5339, concentration of TWR sugar (\%Brix) and short time. The result gave alcohol to $10.00 \%$. This alcohol can be used as the raw materials in industries such as vinegar factory, drinks and fuel factory.

\section{Acknowledgements}

This research was financially supported by the National Science and Technology Development Agency, Ministry of Higher Education, Science, Research and Innovation, Thailand.

\section{References}

1. Varavinit S, Shobsngob S, Varanyanond W, Chinachoti P and Naivikul O 2003 Effect of amylose content on gelatinization, retrogradation and pasting properties of flours from different cultivars of Thai rice Starch/Starke 55(9): 410-415

2. Noosuk P, Hill S E, Pradipasena P and Mitchell J R 2003 Structure-viscosity relationships for Thai rice starches Starch/Starke 55(8): 337-344

3. Ahmad Z S and Munaim M S A 2018 Response surface methodology based optimization of sorbitol production via solid state fermentation process Engineering in Agriculture, Environment and Food

4. Vasiee A, Behbahani B A, Yazdi F T and Moradi S 2016 Optimization of the production conditions of the lipase produced by Bacillus cereus from rice flour through Plackett-Burman Design (PBD) and response surface methodology (RSM) Microbial Pathogenesis 101: $36-43$

5. Chinma C E, Ilowefah M and Muhammad K 2014 Optimization of rice bran fermentation conditions enhanced by baker's yeast for extraction of protein concentrate Nigerian Food Journal 32(1): 126-132

6. Pankaj J, Arup J D, and Sankar C D 2018 Optimisation of fermentation process for production of black rice wine and evaluation of its phenolic and volatile compounds J. Inst. Brew 92: 290-299

7. Francis F, Sabu A, Nampoothiri K M, Ramachandran S, Ghosh S, Szakacs G and Pandey A 2003 Use of response surface methodology for optimizing process parameters for the production of $\alpha$-amylase by Aspergillus oryzae Biochem. Eng. J. 15: 107-115 